\title{
Assessing the adherence behavior of glaucoma patients to topical eye drops
}

\author{
This article was published in the following Dove Press journal: \\ Patient Preference and Adherence \\ 18 December 2014 \\ Number of times this article has been viewed
}

\author{
Ulrich Welge-Lussen' \\ Stefanie Weise ${ }^{2}$ \\ Alice $L \mathrm{Yu}^{3}$ \\ 'Department of Ophthalmology, \\ Friedrich-Alexander University, \\ Erlangen, Germany; ${ }^{2}$ Department \\ of Ophthalmology, University \\ of Cologne, Cologne, Germany; \\ ${ }^{3}$ Department of Ophthalmology, \\ Ludwig Maximilian University, \\ Munich, Germany
}

Correspondence: Alice L Yu

Department of Ophthalmology, LudwigMaximilians University, Mathildenstrasse 8, 80336 Munich, Germany

Tel +4989516038II

Fax +498951605160

Email alice.yu@med.uni-muenchen.de
Purpose: The goal of this study was to determine the adherence of glaucoma patients to their topical glaucoma medication. Furthermore, the relationships between the adherence behavior and the patients' demographic data, clinical characteristics, and their knowledge about glaucoma were evaluated.

Methods: This was a prospective study of 123 patients with primary open-angle glaucoma who were given two standardized questionnaires. The first questionnaire at time point T1 comprised a knowledge assessment and the self-reported adherence measures Adherence to Refills and Medication Scale 2 (ARMS2), visual analogue scale for adherence (VAS-AD), and missed doses in the past 14 days. Two months later at time point $\mathrm{T} 2$, a second questionnaire reevaluated the adherence measures ARMS2, VAS-AD, and missed doses in the past 14 days.

Results: There was a good correlation among all the three adherence measures at T1 and T2. The mean values of ARMS2 were in the lower range, with 3.38 at $\mathrm{T} 1$ and 2.8 at T2. The VAS-AD detected that $18.5 \%$ of patients always took their eye drops correctly, and $77.9 \%$ of patients reported not to have missed a single dose in the past 14 days. There was no significant correlation between the patients' demographic data or knowledge about glaucoma and the adherence measures ARMS2 or VAS-AD. Among the clinical characteristics, only single-eye blindness showed a significant correlation with VAS-AD.

Conclusion: In this study, no general relationships were found between medication adherence and the patients' demographic data, clinical characteristics, or knowledge about glaucoma. It may be assumed that more individualized strategies are required to optimize adherence behavior.

Keywords: adherence, eye drops, glaucoma, medication

\section{Introduction}

Primary open-angle glaucoma (POAG) is a leading cause of blindness in the world. ${ }^{1,2}$ This disease is characterized by elevated intraocular pressure (IOP) and a progressive loss of nerve fibers, leading to optic nerve degeneration. The medical therapy for POAG is based on the regular use of hypotensive eye drops to decrease the IOP. A major determinant for success in medical therapy is the adherence of patients to their medication. ${ }^{3}$ Adherence is defined as the extent to which a patient's behavior in taking medication corresponds with agreed recommendations from a doctor. ${ }^{4}$ In contrast to the term "compliance," adherence requires the patient's agreement to the recommendation. Nonadherence is one of the major problems in glaucoma treatment. The reason is that there are no obvious symptoms in the earlier course for glaucoma patients. ${ }^{5}$ Furthermore, glaucoma medication may have negative side effects. Finally, the glaucoma patient usually requires a lifelong treatment but often does not realize any direct benefits from the therapy. However, nonadherence behavior has longterm impacts on the visual function of the patient. Without hypotensive eye drops, an elevated IOP may lead to progressive optic nerve degeneration and deterioration 
of the visual field. ${ }^{6,7}$ It is estimated that approximately $10 \%$ of visual field defects are caused by nonadherence. ${ }^{6}$ Additionally, nonadherence is associated with improper use of prescribed medication, which may lead to a further burden on our health-economic system. ${ }^{8}$

According to the literature, the rate of nonadherence in glaucoma therapy varies between $5 \%$ and $80 \% .^{5}$ The reason for this great variability of results is the heterogeneity in the measuring devices used and the definitions of adherence. Adherence can be measured by both electronic monitoring devices or by analyses of questionnaires. For instance, the Travatan ${ }^{\mathrm{TM}}$ Dosing Aid Study ${ }^{9}$ demonstrated that the patients' reported adherence rate was much higher than the rate measured by electronic monitoring devices. Furthermore, Konstas et $\mathrm{al}^{6}$ detected that $15 \%$ of glaucoma patients were unconscious of their nonadherence, which meant that the patients were taking their prescribed glaucoma medication at the right time but were unable to apply their eye drops properly. Another possible reason for nonadherence is poor knowledge about the disease. One previous study ${ }^{10}$ postulated that good knowledge about glaucoma may positively influence patients' adherence. Several studies ${ }^{6,10,11}$ showed that many glaucoma patients had little knowledge about their disease. Konstas et $\mathrm{al}^{6}$ demonstrated that only half of their patients knew the definition of glaucoma and only a quarter of their patients were knowledgeable about the fact that glaucoma can potentially lead to blindness.

The goal of the current study was to determine the adherence of glaucoma patients by self-reporting questionnaires. Furthermore, we examined the relationships between the adherence behavior and the patients' demographic data, clinical characteristics, and knowledge about glaucoma. This information may help to identify potential predictors of adherence in glaucoma therapy.

\section{Subjects and methods Design}

A prospective, questionnaire-based study of a consecutive sample of patients with POAG was conducted from April to October 2010 at the University Eye Clinic, Friedrich-Alexander University, Erlangen, Germany. Patients were included in this study if they had had a topical medical therapy for POAG for at least 4 weeks. Exclusion criteria were other types of glaucoma (eg, congenital, closed-angle glaucoma, or secondary glaucoma), eye diseases interfering with glaucoma management, a history of dementia or cognitive restrictions, language barriers, or a disability of reading and writing. At the first point of measurement (T1), the patients were asked to fill in a standardized questionnaire at the University Eye Clinic, Friedrich-Alexander University, Erlangen. Two months later (T2), the patients were requested to answer a second standardized questionnaire at home and send it back to the hospital by mail. The reason to choose the time point T2 as 2 months after T1 was to avoid the influence of scheduled visits to the ophthalmologist on the patients' answers in the questionnaire. In our sample, most glaucoma patients had scheduled their visits every 3 months. This study was performed in accordance with the ethical standards laid down in the 1964 Declaration of Helsinki and was approved by the local ethics committee, Friedrich-Alexander University, Erlangen, Germany. Written informed consent from the patients was obtained for including their data into this study.

In total, 244 patients were considered for participation in this study. Of the 244 patients, 123 patients completed this study until the end of the follow-up period. Of the remaining 121 patients, 40 patients were excluded based on the exclusion criteria, eight patients refused to participate, 60 patients did not complete the questionnaire at $\mathrm{T} 1$, and 13 patients did not answer the questionnaire at T2. POAG was defined as an increase in IOP $\geq 22 \mathrm{mmHg}$ (measured by Goldmann applanation tonometry) and documented optic disk changes and/or visual field defects consistent with glaucoma. Glaucomatous optic disk changes were progressive optic disk cupping, asymmetric optic disk cupping ( $>0.2$ difference), optic disk hemorrhage, acquired pit of the optic nerve, parapapillary retinal nerve fiber layer loss, and large cup-to-disk ratio (thin neuroretinal rim).

Baseline demographic and clinical data were obtained from each patient. The following demographic information was requested from the patients: age, sex, marital status, living situation, educational level, and employment status. Furthermore, the following clinical information was collected from the medical records: first time of diagnosis of glaucoma, current and maximal IOP measured by Goldmann applanation tonometry, best-corrected visual acuity using standardized acuity charts, slit-lamp biomicroscopy, gonioscopy, optic disk changes according to Jonas' classification, ${ }^{12}$ and visual field defects measured by white-on-white Humphrey 30-2 visual tests. The severity of glaucoma was assessed using the clinical data, including visual acuity, visual field defects, and optic disk changes. The extent of visual field defects was assessed according to Mills' classification. ${ }^{13}$ Patients with progressive visual field defects received a Goldmann perimetry and were classified as stage 5 of Mills' classification. The severity of optic disk changes was assessed according to Jonas' classification. The stability of glaucoma 
disease was estimated by the patients' 24-hour IOP profiles, temporal changes in visual field defects, and evaluation by their ophthalmologists.

\section{Measures}

At time point T1, the patients were asked to fill in the first questionnaire, which comprised the following self-reported measures: knowledge assessment, Adherence to Refills and Medication Scale 2 (ARMS2), visual analog scale for adherence (VAS-AD), and missed doses in the past 14 days. Two months later at time point T2, the patients were given a second questionnaire, which reevaluated ARMS2, VAS-AD, and missed doses in the past 14 days.

\section{Assessment of knowledge of glaucoma patients}

The knowledge assessment test was developed according to the studies of Blondeau et $\mathrm{al}^{14}$ and Hoevenaars et al. ${ }^{15}$ The test contained ten specific questions about glaucoma, eg, "Can glaucoma lead to blindness?" or "Does glaucoma affect the visual field?" All questions reflected essential parts of the disease and its therapeutic options and could potentially be answered by glaucoma patients. The answering scale was "no", "yes", and "I do not know".

\section{ARMS score}

The ARMS tool of Kripalani et al ${ }^{16}$ examined adherence to medication in terms of two points: correct form and refill of medication, and correct intake of medication. In particular, the questions were developed in such a way that they were independent of the literacy of the patients. For this study, we created a German-translated and adapted version (ARMS2) of the original version. The validity was determined by correlation with the widely used Morisky scale. The English original version included four items containing prescriptionrefill subscales and eight items containing medication-taking subscales. The 12 items of the original version were answered on a four-point Likert scale, with the answering options "never," "sometimes," "mostly," and "always." We chose a ten-step numeric rating scale, with 0 meaning "never" and 9 meaning "always" for a better differentiation of the answers and adaptation to the remainder of the questionnaire. For the total result of ARMS, all values above zero reflected some degree of nonadherence. The number 1 meant little nonadherence and 99 meant absolute nonadherence, which is equivalent to a rejection of therapy.

The English version of ARMS was validated in the study "Improving medication adherence through graphical enhanced interventions in coronary heart disease" (IMAGE-CHD). ${ }^{16}$ As a criterion of reliability, the authors reported a good internal consistency and test-retest reliability. Cronbach's alpha was 0.81 ; the test-retest reliability was 0.69 .

Adherence assessment by VAS-AD, and missed doses

To assess the adherence of patients to topical glaucoma medication, different question-and-answer formats were developed to decrease the risk of providing distorted information in the self-reporting questionnaires. In a VAS-AD, the patients should indicate how often they took their eye drops as recommended. They were asked to set their answer on a $10 \mathrm{~cm}$ horizontal line, with $0 \mathrm{~cm}$ representing "never" and $10 \mathrm{~cm}$ representing "always." Similar to the questionnaires of Pappa et $\mathrm{al}^{17}$ and Kholdebarin et $\mathrm{al}^{18}$ the patients had to answer the question about how often they forgot the doses in the past 14 days. The patients recorded the application of eye drops. Furthermore, they had to specify the frequency of daily eye drop dosage.

\section{Statistical analysis}

Statistical analyses were conducted with the SPSS software version 18.0 (SPSS; Chicago, IL, USA). Descriptive statistics were used for the calculation of mean values and standard deviations (SDs) at time points T1 and T2. Bivariant correlations were assessed by analyses of dependent and independent variables. Regression models were used to assess the predictability of the independent variables of adherence. Only data with at least one of the adherence measures ARMS2 or VAS-AD were included in the analyses. In subscales with up to eight items, two missing items were accepted. In the subscales with at least eleven items, a maximum of $20 \%$ missing items was accepted. Missing values were substituted by the mean value of the remaining values. $P<0.05$ was regarded as statistically significant.

\section{Results}

\section{Basic demographics and clinical characteristics}

Among a total of 123 patients, there were 78 females $(63.4 \%)$ and 45 males $(36.6 \%)$. The mean age was $67.0 \pm 13.7$ years (range: 45-88 years). Majority of the patients were living with their partners and had retired. Moreover, $74 \%$ of patients $(n=91)$ still lived with their partners, $23.6 \%$ of patients $(n=29)$ had lived in a partnership, and $2.4 \%$ of patients $(n=3)$ were living alone. Concerning the employment status, $70.7 \%$ of patients $(n=87)$ had retired, $12.2 \%$ of patients $(n=15)$ were still full-time employees, $10.6 \%$ of patients $(n=13)$ had parttime employment, and $6.5 \%$ of patients $(n=8)$ had no work. The majority of patients $(55.3 \% ; n=68)$ had a secondary 


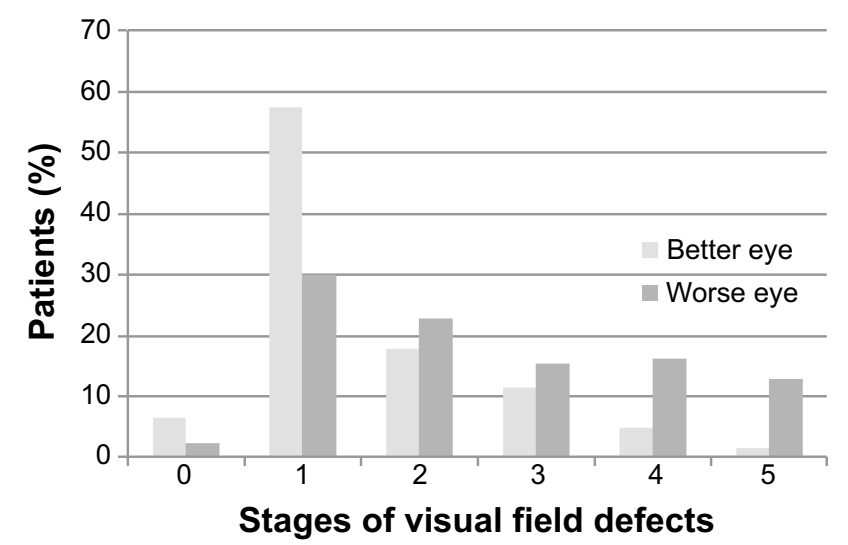

Figure I Stages of visual field defects according to Mills' classification in the study group.

general school certificate, $25.2 \%$ of patients $(n=31)$ had a high school diploma, $13.8 \%$ of patients $(n=17)$ a higher education entrance qualification, and $5.7 \%$ of patients $(n=7)$ had other kinds of educational qualifications.

The mean IOP at T1 was $13.4 \pm 3.1 \mathrm{mmHg}$ (range: $7-28 \mathrm{mmHg}$ ) in the better eye and $15.6 \pm 3.8 \mathrm{mmHg}$ (range: $8-36 \mathrm{mmHg}$ ) in the worse eye. The visual acuity at T1 was $0.8 \pm 0.2$ (range: $0.2-1.2$ ) in the better eye and $0.5 \pm 0.3$ (range: $0.1-1.0$ ) in the worse eye. Blindness in one eye was present in $4.9 \%$ of patients $(n=6)$. The distribution of the visual field defects according to Mills' classification is shown in Figure 1. The distribution of optic disk changes according to Jonas' classification is depicted in Figure 2.

Among the excluded subjects, the mean age of the patients who refused to complete the questionnaire was significantly higher than that of the study participants $(P=0.005)$. There was no significant age difference between the study participants and the patients with incomplete questionnaires (dropouts) $(P=0.743)$. The sex distribution was even in all groups

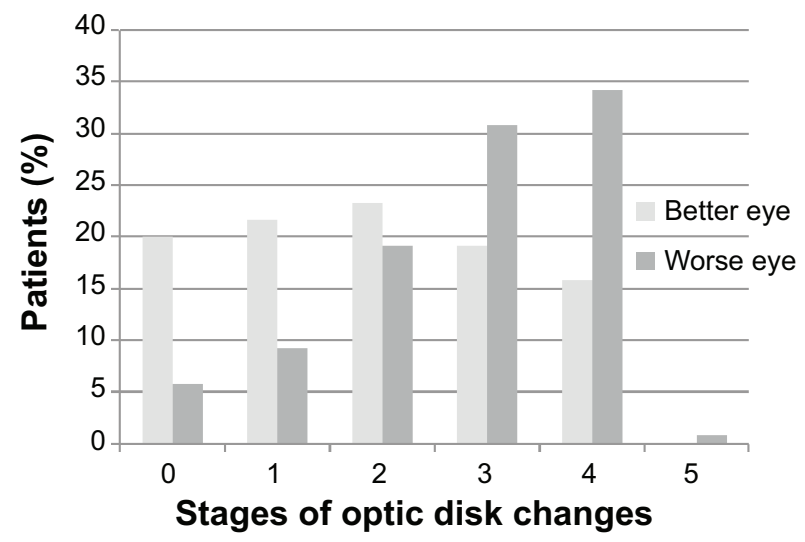

Figure 2 Stages of optic disk changes according to Jonas' classification in the study group.
$(P=0.804)$. The mean IOP of the worse eye of the dropouts was significantly higher than that of the study participants $(P=0.006)$. In contrast, the mean IOP of the better eye was similar in all groups. Patients refusing to fill out the questionnaire had significantly more visual field defects in both the better and the worse eyes $(P=0.004$ and $P=0.005)$. However, the adherence measures were similar between the participants and the nonparticipants at T2 (VAS: $P=0.624$; ARMS: $P=0.064$ ).

\section{Assessment of knowledge of glaucoma patients}

On average, seven out of ten questions in the knowledge assessment questionnaire were answered correctly. However, only $8.2 \%$ of patients answered all questions correctly. More than $95 \%$ of patients knew about the fact that glaucoma can lead to visual field defects and blindness. Approximately half of the patients knew that vision loss is irreversible, although $24.6 \%$ of patients believed that glaucoma could be cured. The most common false assumption made by $80 \%$ of patients was that vision loss could only be caused by an increase in IOP.

\section{Medication adherence}

To determine the medication adherence of glaucoma patients, three adherence measures were studied at both time points T1 and T2: ARMS2, VAS-AD, and missed doses in the past 14 days. The descriptive values of the adherence measure scales are displayed in Table 1. We found a good correlation among all three adherence measures at both time points T1 and T2 (Table 2). The mean values of ARMS2 were in the lower range, with 3.38 at T1 and 2.8 at T2. The mean values in ARMS2 did not significantly vary between $\mathrm{T} 1$ and $\mathrm{T} 2(P=0.335)$. In our study group, the most common reason for nonadherence was forgetfulness, reported by $43.8 \%$ of patients (Figure 3 ). One third of patients missed doses if they had to apply eye drops more than once a day. Inattentiveness as the reason of nonadherence was identified in $25 \%$ of the study participants.

In the VAS-AD, the subjects were asked to indicate how often they took their eye drops correctly. The descriptive

Table I Descriptive values of the adherence measures at $\mathrm{TI}$ and $\mathrm{T} 2$

\begin{tabular}{|c|c|c|c|c|c|c|}
\hline \multirow{2}{*}{$\begin{array}{l}\text { Adherence } \\
\text { measures }\end{array}$} & \multirow[t]{2}{*}{ Possible values } & \multirow[t]{2}{*}{$\mathbf{N}$} & \multicolumn{2}{|l|}{ TI } & \multicolumn{2}{|l|}{ T2 } \\
\hline & & & Mean & SD & Mean & SD \\
\hline ARMS2 & 0-99 & 120 & 3.38 & $(6.89)$ & 2.80 & $(4.05)$ \\
\hline VAS-AD & $0.0-10.0$ & 116 & 9.03 & (I.34) & 9.08 & $(1.03)$ \\
\hline Missed doses & Starting from 0 & 113 & 0.37 & $(0.81)$ & 0.50 & $(1.08)$ \\
\hline
\end{tabular}

Abbreviations: ARMS2, Adherence to Refills and Medication Scale, Germantranslated version; SD, standard deviation; T1, first point of measurement; T2, second point of measurement; VAS-AD, visual analog scale for adherence. 
Table 2 Correlations of the adherence measures at TI and T2

\begin{tabular}{|c|c|c|c|c|c|c|}
\hline \multirow[t]{2}{*}{ Measures, (n) } & \multicolumn{3}{|l|}{ TI } & \multicolumn{3}{|l|}{ T2 } \\
\hline & ( & (2) & (3) & (I) & $(2)$ & (3) \\
\hline ARMS2, (I) & 1 & NA & NA & 1 & NA & NA \\
\hline VAS-AD (P-value), (2) & $-0.4 \mathrm{I}(0.007)$ & 1 & NA & $-0.31(0.006)$ & 1 & NA \\
\hline Missed doses ( $P$-value), (3) & $0.43(0.006)$ & $-0.47(0.007)$ & 1 & $0.53(0.008)$ & $-0.28(0.008)$ & 1 \\
\hline
\end{tabular}

Abbreviations: ARMS2, Adherence to Refills and Medication Scale, German-translated version; NA, not applicable; TI, first point of measurement; T2, second point of measurement; VAS-AD, visual analog scale for adherence.

values of VAS-AD were 9.03 at T1 and 9.08 at T2 (Table 1). They were similar between $\mathrm{T} 1$ and $\mathrm{T} 2(P=0.735)$. At T1, $18.5 \%$ of patients indicated that they always took the eye drops correctly; $68.9 \%$ of patients had a correct intake in at least $90 \%$ of occasions.

The average missed doses in the past 14 days showed the lowest variance: $77.9 \%$ of patients indicated that they did not miss a single dose in the past 14 days. Two months later, $74.2 \%$ of patients did not miss a single dose in the past 14 days. The mean number of missed doses at T1 was 0.37 doses in the past 14 days. At T2, the patients had missed a mean of 0.50 doses in the past 14 days (Table 1 ). These values were also stable during the follow-up period $(P=0.110)$.

\section{Correlation between demographic/ clinical characteristics and adherence}

To analyze whether demographic or clinical characteristics influence the adherence behavior of glaucoma patients, we correlated them with ARMS2 and VAS-AD at both time points T1 and T2 (Table 3). For the demographic data, we did not find any significant correlations between the patients' age $(P=0.1)$, sex $(P=0.23)$, or educational level $(P=0.21)$ and the adherence measures ARMS2 or VAS-AD. Among the clinical characteristics, only blindness showed a significant correlation with VAS-AD $(P=0.008)$. Single-eye blindness was associated with fewer correct applications of eye drops. Other clinical parameters such as IOP, visual acuity, visual field defects, and optic disk changes did not correlate with ARMS2 or VAS-AD.

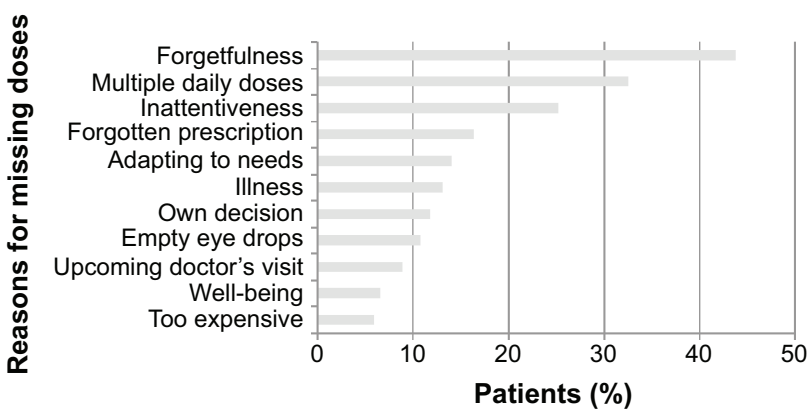

Figure 3 Reasons for missing doses of eye drops.

\section{Correlation between knowledge of glaucoma and adherence}

The descriptive values of the knowledge assessment only showed a weak correlation with the adherence measures ARMS2 and VAS-AD at both time points T1 (ARMS2: -0.08; VAS-AD: -0.05) and T2 (ARMS2: -0.07; VAS-AD: 0.04).

\section{Discussion}

In this study, we investigated patients' adherence to topical eye drops by using three different adherence measures. There was a good correlation among ARMS2, VAS-AD, and missed doses in the past 14 days. Therefore, it can be assumed that all three measures assessed the same construct of adherence. The descriptive values of all three measures demonstrated that most patients in our study group were adherent to their topical glaucoma medication. There were no significant changes in the adherence behavior between the first evaluation at hospital (T1) and the second one 2 months later at home (T2). We chose the time interval of 2 months between time points $\mathrm{T} 1$ and $\mathrm{T} 2$ because we wanted to avoid the influence of the eye doctor's visits on the adherence behavior. In a study by Kass et $\mathrm{al}^{19}$ it could be demonstrated that there was a significant increase in the adherence rate 24 hours before an appointment with the ophthalmologist. Unfortunately, many of our patients reported paying a visit to their ophthalmologist right after answering the second questionnaire. Therefore, it would have been better to set the time point $\mathrm{T} 2$ in a more individualized time schedule. This would have helped obtain adherence data independent of a doctor's appointment. Further studies are certainly required to observe the adherence behavior of glaucoma patients during a long-term follow-up period.

In previous studies, adherence barriers were divided into patient-related, disease-related, and therapy-related factors. The patient-related factors include demographic data of the patient such as sex or educational level. Previously, a significant correlation was detected between the male sex and nonadherence. ${ }^{6,20}$ Kholdebarin et $\mathrm{al}^{18}$ found a positive association between low educational level and nonadherent behavior. In this study, we could not detect any significant 
Table 3 Correlations between demographic/clinical factors and adherence

\begin{tabular}{|c|c|c|c|c|}
\hline & \multicolumn{2}{|l|}{ TI } & \multicolumn{2}{|l|}{ T2 } \\
\hline & ARMS2 & VAS-AD & ARMS2 & VAS-AD \\
\hline \multicolumn{5}{|l|}{ Demographic factors } \\
\hline Age & -0.13 & 0.09 & -0.14 & 0.04 \\
\hline Sex & -0.01 & -0.06 & 0.01 & 0.05 \\
\hline Education & -0.08 & 0.08 & -0.07 & 0.03 \\
\hline \multicolumn{5}{|l|}{ Clinical factors } \\
\hline IOP & 0.01 & -0.01 & -0.09 & 0.15 \\
\hline Maximal IOP & -0.09 & 0.07 & -0.13 & -0.13 \\
\hline Visual acuity & -0.03 & -0.03 & 0.05 & -0.02 \\
\hline Stage of visual field defects & 0.04 & 0.09 & -0.09 & 0.04 \\
\hline Stage of optic disk changes & -0.01 & 0.04 & -0.09 & -0.01 \\
\hline Blindness & 0.12 & $-0.27 * *$ & 0.01 & -0.05 \\
\hline
\end{tabular}

Notes: $* * P<0.01$.

Abbreviations: ARMS2, Adherence to Refills and Medication Scale, German-translated version; IOP, intraocular pressure; TI , first point of measurement; T2, second point of measurement; VAS-AD, visual analog scale for adherence.

correlations between demographic factors and the adherence behavior of our study participants. One reason for this might be the differences in patient population. The patients in our study had a long history of glaucoma and were relatively well informed. Therefore, demographic factors might have less influence on the adherence behavior in our specific study group. Disease-related adherence barriers include visual acuity, visual field defects, and blindness. A previous study ${ }^{10}$ has shown that patients were more adherent to medication if they had worse visual field defects or were blind in one eye. However, blindness in both eyes apparently reduced the adherence rate because both-eye-blinded patients are probably more dependent on the help of a third person. ${ }^{21}$ In this study, single-eye blindness was associated with worse adherence values as measured by VAS-AD. A relationship between the severity of disease and the adherence behavior was also previously described. ${ }^{22}$ In particular, the severity of glaucoma was assessed using clinical parameters such as visual acuity, visual field defects, and optic disk changes. We could not detect any significant correlation between visual acuity, visual field defects, or optic disk changes and adherence behavior. However, most of our patients had only early stages of glaucoma, so that few patients of our study group were actually affected by severe visual impairment. Therefore, it remains unclear whether these clinical parameters may influence the adherence behavior of glaucoma patients in general.

Another factor that may influence the adherence behavior is the patients' knowledge about their disease. In the 1970 s, Spaeth ${ }^{10}$ observed that patients took their eye drops less often in a correct manner if they were insufficiently knowledgeable about glaucoma. This observation could not be confirmed in the current study. However, it must be taken into account that our patients represented a well-informed group. More than $90 \%$ of our study participants had good knowledge about glaucoma. Therefore, an increase in knowledge would probably have no additional influence on the adherence behavior. However, our results could not predict whether patients' knowledge about glaucoma may affect the adherence behavior in other glaucoma patients. Hoevenaars et al ${ }^{15}$ also did not find a significant correlation between patients' knowledge about glaucoma and their adherence behavior. They even showed that knowing the fact that glaucoma is a slowly progressive disease was associated with lower adherence to the medication. Until now, no clear correlation between patients' knowledge of glaucoma and their adherence behavior has been demonstrated. ${ }^{15}$

One limitation of our study was to have excluded glaucoma patients who did not meet the inclusion criteria. Besides that, our results relied on self-reporting questionnaires of glaucoma patients, who may tend to give socially compatible answers. Other limitations were the small sample size of patients and the period between $\mathrm{T} 1$ and $\mathrm{T} 2$. In addition, environmental factors, social support, and overall medication regimen were also not identified. Furthermore, no second group of participants was added as a control group to this study. Finally, the questionnaire was only administered to patients of a university hospital, which may not represent the general population of patients with glaucoma. Therefore, studies that are more extensive with greater sample sizes, longer assessment period, and objective measurements of adherence are planned in the future.

In summary, we could not show any strong influence of the patients' demographic data, their clinical characteristics, or their knowledge about glaucoma on their medication adherence. Probably, there are no general rules and more individualized strategies are required to optimize adherence behavior. Individualized patient care already has the potential to improve adherence to glaucoma therapy. ${ }^{23}$ Therefore, it will become more important to assess the health-care needs and patient's views on glaucoma before prescribing antiglaucomatous eye drops. Furthermore, this study also demonstrates the difficulty in measuring the adherence rate to medication independent of doctor's appointments. Assuming that doctor's visits may influence medication adherence, a broader use of reminder systems such as phone call reminders may help to improve patients' adherence behavior. 


\section{Acknowledgment}

The data presented in this study are part of the medical thesis of Stefanie Weise at the Medical School of the Friedrich-Alexander University, Erlangen, Germany.

\section{Disclosure}

The authors report no conflicts of interest in this work.

\section{References}

1. Quigley HA. Number of people with glaucoma worldwide. $\mathrm{Br} J \mathrm{Oph}$ thalmol. 1996;80:389-393.

2. Quigley HA, Broman AT. The number of people with glaucoma worldwide in 2010 and 2020. Br J Ophthalmol. 2006;90:262-267.

3. Weinreb RN, Aung T, Medeiros FA. The pathophysiology and treatment of glaucoma: a review. JAMA. 2014;311:1901-1911.

4. WHO. Adherence to Long-Term Therapies: Evidence for Action. Geneva: World Health Organization; 2003.

5. Olthoff CMG, Schouten JSAG, van de Borne BW, Webers CAB. Noncompliance with ocular hypotensive treatment in patients with glaucoma or ocular hypertension an evidence-based review. Ophthalmology. 2005; 112:953-961.

6. Konstas AGP, Maskaleris G, Gratsonidis S, Sardelli C. Compliance and viewpoint of glaucoma patients in Greece. Eye. 2000;14:752-756.

7. Stewart WC, Konstas AGP, Pfeiffer N. Patient and ophthalmologist attitudes concerning compliance and dosing in glaucoma treatment. J Ocular Pharmacol Ther. 2004;20:461-469.

8. Reeder CE, Franklin M, Bramley TJ. Managed care and the impact of glaucoma. Am J Manag Care. 2008;14:S5-S10.

9. Okeke CO, Quigley HA, Jampel HD, et al. Adherence with topical glaucoma medication monitored electronically the Travatan Dosing Aid study. Ophthalmology. 2009;116:191-199.

10. Spaeth GL. Visual loss in a glaucoma clinic. I. Sociological considerations. Invest Ophthalmol. 1970;9:73-82.

11. Bour T, Blanchard F, Segal A. Observance the' rapeutique et ve' cu du glaucome primitif a' angle ouvert. A propos de 341 cas dans la Marne [Therapeutic observance and life of patients with primary openangle glaucoma. Apropos of 341 cases in the department of Marne] J Fr Ophtalmol. 1993;16:380-391. French.
12. Jonas JB, Gusek GC, Naumann GOH. Die parapapilläre region in normal- und Glaukomaugen [The parapapillary region of normal and glaucoma eyes]. Klin Monbl Augenheilkd. 1988;193:52-61. German.

13. Mills RP, Budenz DL, Lee PP, et al. Categorizing the stage of glaucoma from pre-diagnosis to end-stage disease. Am J Ophthalmol. 2006; 141:24-30.

14. Blondeau P, Esper P, Mazerolle E. An information session for glaucoma patients. Can J Ophthalmol. 2007;42:816-820.

15. Hoevenaars JGMM, Schouten JSAG, van den Borne B, Beckers HJM, Webers CAB. Will improvement of knowledge lead to improvement of compliance with glaucoma medication? Acta Ophthalmol. 2008;86: 849-855.

16. Kripalani S, Risser J, Gatti ME, Jacobson TA. Development and evaluation of the Adherence to Refills and Medications Scale (ARMS) among low-literacy patients with chronic disease. Value Health. 2009;12: 118-123.

17. Pappa C, Hyphantis T, Pappa S, et al. Psychiatric manifestations and personality traits associated with compliance with glaucoma treatment J Psychosom Res. 2006;61:609-617.

18. Kholdebarin R, Campbell RJ, Jin Y-P, Buys YM. Multicenter study of compliance and drop administration in glaucoma. Can J Ophthalmol. 2008;43:454-461.

19. Kass MA, Meltzer DW, Gordon M. Compliance with topical pilocarpine treatment. Am J Ophthalmol. 1986;101:515-523.

20. Bloch S, Rosenthal AR, Friedman L, Caldarolla P. Patient compliance in glaucoma. Br J Ophthalmol. 1977;61:531-534.

21. Vincent PA. Patient's viewpoint of glaucoma. Sight Sav Rev. 1972;42: 213-221.

22. Sleath B, Blalock S, Covert D, et al. The relationship between glaucoma medication adherence, eye drop technique, and visual field defect severity. Ophthalmology. 2011;118:2398-2402.

23. Gray TA, Fenerty C, Harper R, et al. Individualised patient care as an adjunct to standard care for promoting adherence to ocular hypotensive therapy: an exploratory randomised controlled trial. Eye (Lond). 2012; 26:407-417.
Patient Preference and Adherence

\section{Publish your work in this journal}

Patient Preference and Adherence is an international, peer-reviewed, open access journal that focuses on the growing importance of patient preference and adherence throughout the therapeutic continuum. Patient satisfaction, acceptability, quality of life, compliance, persistence and their role in developing new therapeutic modalities and compounds to optimize

\section{Dovepress}

clinical outcomes for existing disease states are major areas of interest for the journal. This journal has been accepted for indexing on PubMed Central. The manuscript management system is completely online and includes a very quick and fair peer-review system, which is all easy to use. Visit http://www. dovepress.com/testimonials.php to read real quotes from published authors. 